\title{
Tissue Expression of Low and High Molecular Weight Cytokeratins in Lung Carcinoma Sections: Its Correlation with Some Clinic-Pathological Features
}

\author{
Charles E. Rengifo, ${ }^{1}$ Rancés Blanco, ${ }^{2}$ Mercedes Cedeño, ${ }^{2}$ \\ Milagros Frómeta, ${ }^{2}$ and Enrique Rengifo ${ }^{2}$ \\ ${ }^{1}$ Department of Pathology, Manuel Fajardo Teaching Hospital, Zapata and D Street Vedado, \\ Plaza de la Revolución, 10400 Havana, Cuba \\ ${ }^{2}$ Laboratory of Specific Recognition and Biological Activity, Department of Quality Control, \\ Center of Molecular Immunology, 216 Street and 15 Avenue, Atabey, Playa, P.O. Box 16040, 11600 Havana, Cuba
}

Correspondence should be addressed to Rancés Blanco; rances@cim.sld.cu

Received 8 September 2014; Accepted 30 October 2014; Published 17 November 2014

Academic Editor: Francesco Cappello

Copyright (C) 2014 Charles E. Rengifo et al. This is an open access article distributed under the Creative Commons Attribution License, which permits unrestricted use, distribution, and reproduction in any medium, provided the original work is properly cited.

\begin{abstract}
The tissue expression of low (LMW) and high (HMW) molecular weight cytokeratins and Ber-EP4 antigen in both small (SCLC) and non-small (NSCLC) cell lung carcinomas, as well as its correlation with a variety of clinic-pathological features, was evaluated. In general, 43/52 (82.7\%) of NSCLC sections showed the expression of at least one type of cytokeratin while only 7/16 (43.7\%) of SCLC were stained with both LMW cytokeratin and pan-cytokeratins antibodies. Remarkably, 18/52 (34.6\%) of NSCLC were positive to both types of cytokeratins. However, none of SCLC showed this pattern of expression. In NSCLC patients, the increasing levels of HMW cytokeratins expression, as shown by $34 \beta \mathrm{E} 12$ antibody, correlated with the occurrence of disease recurrence $(P=$ 0.0057; Fisher's exact test). Consequently, the expression of HMW cytokeratins was found to be associated with a poor 4-year overall survival of NSCLC patients $(P=0.0315$; Log rank test), not taking into account the histopathological classification of tumors. Similar results were obtained when 8-year overall survival was assessed $(P=0.0103$; $\log$ rank test). Our results could suggest the assessment of HMW cytokeratins in a larger series of NSCLC samples in order to confirm the potential prognostic value of them.
\end{abstract}

\section{Introduction}

Lung cancer is the most frequent cancer in the world and usually has a very poor prognosis $[1,2]$. There are two main variants of the disease, non-small cell lung cancer (NSCLC) and small cell lung cancer (SCLC). But, NSCLC constitutes about $80-85 \%$ cases of all lung carcinomas $[3,4]$. NSCLC describes a histologically heterogeneous group of tumors with variable clinical behavior. Disease stage and performance status at time of diagnosis have been considered the two most noticeable prognostic factors in patients with NSCLC [5]; however, clinical outcomes in individual patients remain imprecise [6].

Consequently, novel biomarkers are currently assessed for early diagnosis and predicting treatment response and prognosis in NSCLC patients. Between them, markers originating from cytoskeleton, especially the cytokeratins (CK), are of great practical interest to the clinicians and researchers [7]. CKs are the intermediate filament-forming proteins of epithelial cells, although they are expressed in a tissuespecific manner in normal organs and tumors that arise from them. Two types of cytokeratins are distinguished that form heterodimers, namely, acidic type I (cytokeratins 9-19) and basic to neutral type II (cytokeratins 1-8). Cytokeratins may also be classified as low molecular weight (commonly 8, 18, and 19) and high molecular weight (commonly 1, 5, 10, and 14) $[8]$.

Due to the fact that epithelial tumors largely maintain the features of specific keratin expression associated with the respective cell type of origin, keratins have long and 
extensively been used as immunohistochemical markers in diagnostic tumor pathology [9]. Since the development of monoclonal antibodies against several of the CK polypeptides, they are used to evaluate the pattern of CK expression in a variety of epithelial tumors [10] permitting the identification of particular types of cell differentiation and becoming useful diagnostic tools [9].

Additionally, these molecules have been considered important protectors of epithelial structural integrity under conditions of stress, regulators of other cellular functions, including motility, signaling, growth, and protein synthesis [8]. Moreover, accumulating evidence points to their importance as prognostic markers and, more interestingly, as active regulators of epithelial tumorigenesis and treatment responsiveness [8]. For that reason, in this study the tissue expression of both low and high molecular weight cytokeratin in lung carcinoma sections, as well as its association with a variety of clinic-pathological features, was evaluated. In addition, the expression of a transmembrane glycoprotein functioning as a cellular adhesion molecule (Ber-EP4) was assessed in the same samples.

\section{Materials and Methods}

2.1. Monoclonal Antibodies. Monoclonal antibodies against high molecular weight cytokeratins (1, 5, 10, and 14) (clone $34 \beta$ E12, Dako) and low molecular weight cytokeratins (cytokeratin 8) (clone 35BH11, Dako) were used. Additionally, the pan-cytokeratins (clone MNF116, Dako) that react with cytokeratins 5, 6, 8, 17, and probably also 19 as well as a monoclonal antibody that recognize a transmembrane glycoprotein functioning as a cellular adhesion molecule, Ber-EP4 (clone Ber-EP4, Dako), were also evaluated. All Mabs were diluted 1:20 using an antibody diluent solution (Dako S0809).

2.2. Tissue Specimens. A number of 52 and 16 routinely processed formalin-fixed and paraffin-embedded archival samples with diagnosis of non-small cell carcinoma and small cell carcinoma, respectively, were taken from the pathology department of both Hermanos Ameijeiras General Hospital and the National Institute of Oncology and Radiobiology, after obtaining approved consent by the institutional ethical committees.

2.3. Pathological Features Evaluation. Some morphological parameters such as histopathological classification, grade of differentiation, degree of cellular pleomorphism, and mitotic and necrosis indexes were evaluated for an expert pathologist (ChER) in each section using hematoxylin and eosin (H/E) staining as described in [11]. Briefly, the degree of cell pleomorphism was evaluated considering the cytomorphologic characteristics of tumor cells and it was grouped as low and high degree cell pleomorphism. Mitotic index was recorded by the evaluation of 10 high-power fields with $400 \mathrm{x}$ magnification (10x ocular, 40x objective) and it was calculated by dividing cells out of total cells counted and expressed as previously described for cell pleomorphism. For the evaluation of the degree of tumor necrosis (necrosis index) on each section a low-power field with $100 \mathrm{x}$ magnification $(10 \mathrm{x}$ ocular, 10x objective) was used. It was scored subjectively as follows: low necrotic index (less than $50 \%$ of necrosis areas per field) and high necrotic index (more than $50 \%$ of necrosis areas per field).

2.4. Immunohistochemical Staining. Five micrometer serial sections from each paraffin block were obtained in a micrometer (Leitz 1512) and they were mounted on plus slides (Dako S2024). All sections were attached to the slide by heating in a $70^{\circ} \mathrm{C}$ oven for $1 \mathrm{~h}$. Afterwards the slides were kept at room temperature until they were used.

The slides were dewaxed in xylene and rehydrated in graded ethanol series as usual and endogenous peroxidase activity was blocked with $0.03 \%$ hydrogen peroxide in absolute methanol for 30 minutes. All sections were rehydrated in tap water, distilled water, and Tris/saline buffer solution (TBS) for 15, 10, and 5 minutes, respectively. The slides were pretreated with $0.4 \%$ pepsin in $0.1 \mathrm{~N}$ hydrochloric acid solution at $37^{\circ} \mathrm{C}$ for 30 minutes. After pretreatments, the slides were washed gently in tap water and then with distilled water and TBS, as were described above.

Afterward, the tissues were incubated with primary monoclonal antibodies (see Section 2.1) in a humid chamber for $1 \mathrm{~h}$ at room temperature. After two rinses in TBS, the slides were incubated at room temperature with a rabbit antimouse biotinylated secondary antibody (Dako E0354) and ABComplex/HRP (Dako E0355) during 30 minutes both at a dilution 1:100. Between incubations, slides were washed with TBS for 10 minutes. Afterward, enzymatic activity was visualized with DAB substrate chromogenic solution (Dako K3465) and the tissues were counterstained with Mayer's hematoxylin (Dako S2020). The samples were dehydrated and mounted with a synthetic medium. The analysis was performed using an Olympus BX51 bright-field microscope.

For conventional fluorescent and confocal microscopies, after the incubation with the primary antibody, a rabbit antimouse fluoresceinated secondary antibody (Dako E0354) (green signal) was used and finally the nuclei were counterstained with propidium iodide (PI) (Sigma P4170) at a final concentration of $4 \mu \mathrm{g} / \mathrm{mL}$ (red signal). The analysis was performed using a Leica DMRXA fluorescence microscope and a Leica SP2 confocal microscope.

2.5. Immunohistochemical Evaluation. All tissue markers were evaluated for percentage of positive cells (0-100\%) and the intensity of reaction $(0-3+)$. The final results were considered according to two observers agreement (ChER and $\mathrm{RB})$. Afterward, the IHC score (H-score) was used as was previously described [12] with minor modifications. The $\mathrm{H}$ score was calculated for each specimen by multiplication of the intensity of reaction and the grade of positive cells, resulting in a score ranging from 0 to 300. Subsequently, these scores were grouped as follows: low expression (scores $<150$ ) and high expression (scores $\geq 150$ ).

2.6. Statistical Analysis. GraphPad Prism 5 Software (2007 GraphPad Software Inc., La Jolla, CA, USA) was used for data 
analysis. Survival distribution was estimated by the KaplanMeier method. Two-sided Log-rank tests were performed to compare overall survival (OS) distribution according to cytokeratins and Ber-EP4 expressions as well as the clinicpathological features. Chi-square and Fisher's exact tests were used to compare two or three different parameters. The correlation between biomarkers and clinic-pathological features was assessed by Spearman ranks correlation coefficients. For all tests, the criterion for statistical significance was $P<0.05$.

\section{Results}

3.1. Patient Description and Pathological Features. Table 1 shows a summary of patient characteristics and some pathological features. The gender ratio was close to $2: 1$ in favor of males, with a mean age of 55.0 years (range $23-86$ years). In general, the 4-year overall rate of NSCLC patients was $75.0 \%$ $(29 / 52)$ while the mean overall survival of this population was 37.6 months (ranged from 3.0 to 54.3). Concerning SCLC, the overall survival was $56.3 \%$ (9/16) with a mean of 25.9 months (ranged from 0.9 to 41.6). Statistically significant difference was obtained when the survival of SCLC patients was compared with that from NSCLC $(P=0.0478$; Log rank test).

When disease recurrence, grade of tumor differentiation, and both necrosis and mitotic index were compared between NSCLC and SCLC statistically significant differences were obtained $(P=0.0216, P=0.0001, P=0.0009$, and $P=$ 0.0006; Fisher's exact test, resp.). Tumor stage as well as the occurrence of both lymph node and disease recurrence was associated with poorer overall survival of NSCLC patients $(P=0.0372, P=0.0140$, and $P=0.0024$; Log rank test, resp.). In addition, a tendency of association between the appearance of other site metastasis and overall survival of patients was detected ( $P=0.0522$; Log rank test, resp.).

3.2. Distribution of Cytokeratins in NSCLC and SCLC Samples. Table 2 shows the tissue expression of cytokeratins according to the histopathological classification of tumors. In general, $43 / 52(82.7 \%)$ of NSCLC sections showed the expression of at least one type of cytokeratin. The expression of these molecules was mainly evidenced in the cytoplasm of malignant epithelial cells, although the cell membrane was also decorated (Figure 1). According to cytokeratin types, $35 / 52(67.3 \%)$ of NSCLC were positive with pan-cytokeratins antibody, 37/52 (71.1\%) expressed low molecular weight cytokeratin, and 22/52 (42.3\%) expressed high molecular weight cytokeratin (Figure 2). No expression of high molecular weight cytokeratins was detected in SCLC (0/16) while $7 / 16(43.7 \%)$ of cases were stained with both low molecular weight cytokeratin and pan-cytokeratins antibodies.

When the reactivity of high molecular weight cytokeratin in NSCLC and SCLC was compared statistically significant differences were evidenced ( $P=0.0015$, Fisher's exact test). No differences were detected when the reactivity of low molecular weight cytokeratin and pan-cytokeratins antibodies was compared in these types of tumors $(P=0.4001$ and $P=0.1403$, Fisher's exact test; resp.). No significant differences were observed when the expression of high molecular weight cytokeratins was compared in epidermoid carcinomas, adenocarcinomas, and large cell carcinomas ( $P=0.1045$, Chi-square test). Similar results were obtained with low molecular weight cytokeratin and pan-cytokeratins Mabs ( $P=0.9505$ and $P=0.6969$, Chi-square test; resp.).

As expected, the expression of high and low molecular weight cytokeratins correlates with the reactivity of pancytokeratins monoclonal antibody $(P=0.0366$, $r s=0.2906$, and $P<0.0001, r s=0.7715$; Spearman test). Nevertheless, the expression of high molecular weight cytokeratin tended to correlate with the expression of low molecular weight cytokeratin $(P=0.0929, r s=0.2354$; Spearman test $)$.

\subsection{Considerable Overlapping of Cytokeratins Was Evidenced} in NSCLC but Not in SCLC. Table 3 shows the tissue expression of both high and low molecular weight cytokeratins according to the histopathological classification of tumors. In general, $18 / 52(34.6 \%)$ of NSCLC were positive to both types of cytokeratins. However, none of SCLC (0/16) showed this pattern of expression. Large cell carcinoma displayed the higher rate of cytokeratins overlapping (5/10) followed by epidermoid carcinomas (7/16) and the other histological type minority represented 2/6. Concerning adenocarcinomas, these tumors showed the lower rate of cytokeratins overlapping $(4 / 20)$.

3.4. Ber-EP4 Expression Correlates with Low Molecular Weight Cytokeratin in Both NSCLC and SCLC Patients. The tissue expression of Ber-EP4 according to the histopathological type is shown in Table 4. In general, the expression of this antigen was evidenced in 33/52 (63.5\%) and 7/16 (43.7\%) of NSCLC and SCLC, respectively. No differences were detected when the expression of Ber-EP4 was compared in this group of tumors $(P=0.5678$; Fisher's exact test). Significant differences were found when the expression of Ber-EP4 in adenocarcinoma was compared with epidermoid carcinomas and large cell carcinomas $(P=0.0002$ and $P=0.0004$; Fisher's exact test, resp.). No differences were detected when the expression of this antigen in epidermoid and large cell carcinomas was compared ( $P=0.8876$; Fisher's exact test).

On the other hand, the expression of Ber-EP4 antigen in NSCLC samples correlates with the expression of low molecular weight cytokeratin $(P=0.0113, r s=0.3485$; Spearman test). A tendency to correlate with pan-cytokeratins expression $(P=0.0597, r s=0.2629$; Spearman test $)$ was also evidenced, while no correlation with the expression of high molecular weight cytokeratin was detected $(P=0.5631$, $r s=$ 0.0820; Spearman test). In SCLC, a statistically significant correlation was obtained when the expression of Ber-EP4 antigen was compared with the reactivity of low molecular weight and pan-cytokeratins antibodies $(P=0.0009$, $r s=$ 0.7460; Spearman test, in both cases).

\subsection{The Expression of High Molecular Weight Cytokeratins} Was Associated with Disease Recurrence in NSCLC Patients. In order to correlate the expression of cytokeratins with the clinic-pathological features, the expression of these molecules 
TABLE 1: Clinic-pathological characteristics.

\begin{tabular}{|c|c|c|c|}
\hline Features & $\begin{array}{c}\text { NSCLC }(n=52) \\
\text { Number of cases (\%) }\end{array}$ & $\begin{array}{c}\text { SCLC }(n=16) \\
\text { Number of cases }(\%)\end{array}$ & $P$ value \\
\hline \multicolumn{4}{|l|}{ Gender } \\
\hline Female & $15(28.8)$ & $6(37.5)$ & \multirow{2}{*}{0.5460} \\
\hline Male & $37(71.2)$ & $10(62.5)$ & \\
\hline \multicolumn{4}{|l|}{ Age } \\
\hline$<60$ & $37(71.2)$ & $13(81.2)$ & \multirow{2}{*}{0.5294} \\
\hline$\geq 60$ & $15(28.8)$ & $3(18.8)$ & \\
\hline \multicolumn{4}{|l|}{ Tumor size $(\mathrm{cm})$} \\
\hline$<3$ & $12(23.1)$ & $7(43.7)$ & \multirow{2}{*}{0.1227} \\
\hline$\geq 3$ & $40(76.9)$ & $9(56.3)$ & \\
\hline \multicolumn{4}{|l|}{ Tumor stage } \\
\hline Limited disease & - & $13(81.2)$ & \multirow{4}{*}{-} \\
\hline Extended disease & - & $3(18.8)$ & \\
\hline I-IIIA & $44(84.6)$ & - & \\
\hline IIIB-IV & $8(15.4)$ & - & \\
\hline \multicolumn{4}{|l|}{ Recurrence } \\
\hline Yes & $18(34.6)$ & $11(68.7)$ & \multirow{2}{*}{0.0216} \\
\hline No & $34(65.4)$ & $5(31.3)$ & \\
\hline \multicolumn{4}{|l|}{ Lymph node metastasis } \\
\hline Yes & $17(32.7)$ & $2(12.5)$ & \multirow{2}{*}{0.2012} \\
\hline No & $35(67.3)$ & $14(87.5)$ & \\
\hline \multicolumn{4}{|l|}{ Other site metastases } \\
\hline Yes & $5(9.6)$ & $2(12.5)$ & \multirow{2}{*}{0.6640} \\
\hline No & $47(90.4)$ & $14(87.5)$ & \\
\hline \multicolumn{4}{|l|}{ Grade of differentiation } \\
\hline Well/moderate & $32(61.5)$ & $1(6.2)$ & \multirow{2}{*}{0.0001} \\
\hline Poor/undifferentiated & $20(38.5)$ & $15(93.8)$ & \\
\hline \multicolumn{4}{|c|}{ Degree of cell pleomorphism } \\
\hline Low & $25(48.1)$ & $7(43.7)$ & \multirow{2}{*}{0.7834} \\
\hline High & $27(51.9)$ & $9(56.3)$ & \\
\hline \multicolumn{4}{|l|}{ Necrosis index } \\
\hline$<50 \%$ & $38(73.1)$ & $4(25.0)$ & \multirow{2}{*}{0.0009} \\
\hline$\geq 50 \%$ & $14(26.9)$ & $12(75.0)$ & \\
\hline \multicolumn{4}{|l|}{ Mitotic index } \\
\hline Low & $24(46.2)$ & $0(0)$ & \multirow{2}{*}{0.0006} \\
\hline High & $28(53.8)$ & $16(100)$ & \\
\hline
\end{tabular}

NSCLC, non-small cell lung carcinoma; SCLC, small cell lung carcinoma; \%, percentages.

TABLE 2: Cytokeratins profile expression according to histopathological type.

\begin{tabular}{lcccccc}
\hline Anti-CKs antibodies & $n$ & \multicolumn{2}{c}{$34 \beta{\mathrm{E} 12^{\mathrm{a}}}^{\mathrm{a}}$} & \multicolumn{2}{c}{$35 \mathrm{BH} 11^{\mathrm{b}}$} & MNF116 $^{\mathrm{c}}$ \\
\hline Low/high expression (\%) & & - & + & - & + & + \\
Epidermoid carcinoma & 16 & 50.0 & 50.0 & 25.0 & 75.0 & 25.0 \\
Adenocarcinoma & 20 & 75.0 & 25.0 & 25.0 & 75.0 & 25.0 \\
Large cell carcinoma & 10 & 40.0 & 60.0 & 30.0 & 70.0 & 40.0 \\
Others & 6 & 50.0 & 50.0 & 50.0 & 50.0 & 45.0 \\
Small cell carcinoma & 16 & 0 & 0 & 56.3 & 43.7 & 60.0 \\
\hline
\end{tabular}

a $34 \beta$ E12 reacts with high molecular weight cytokeratins $(1,5,10$, and 14$) ;{ }^{b} 35 B H 11$ stains cytokeratin 8 (low molecular weight cytokeratin); ${ }^{\mathrm{c}} \mathrm{MNF116}$ reacts with cytokeratins 5, 6, 8, 17, and probably also 19 . 


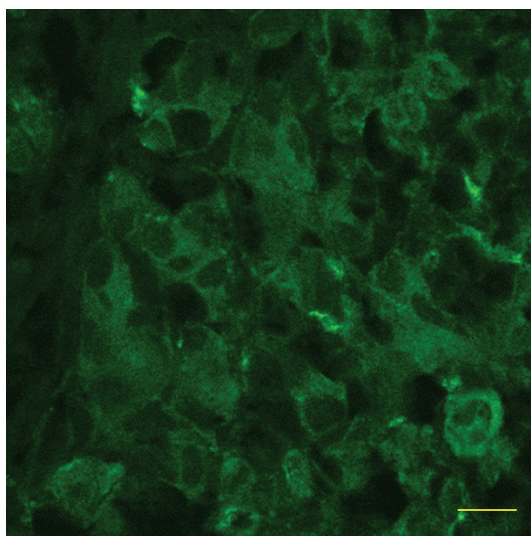

(a)

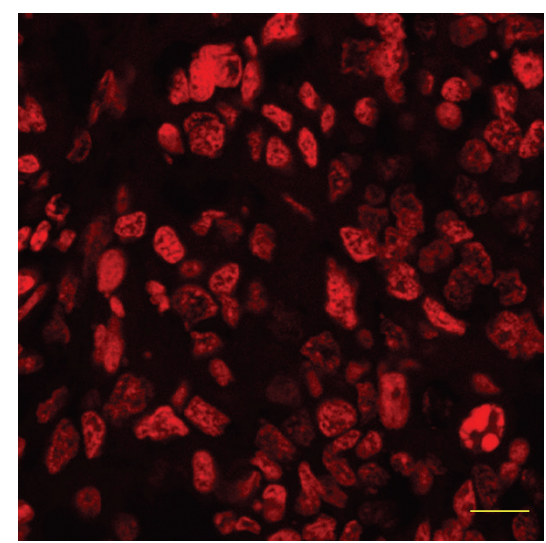

(b)

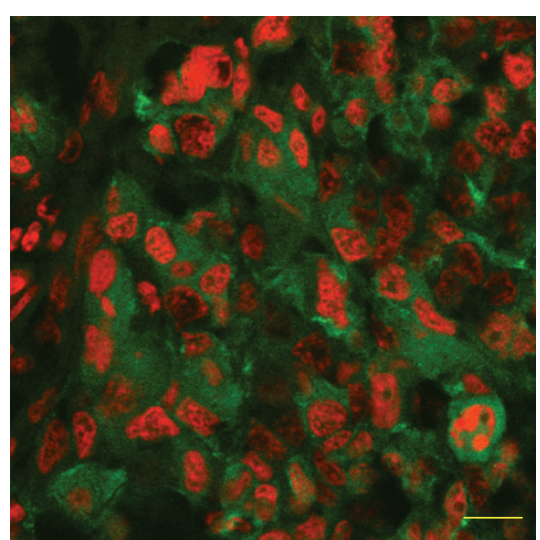

(c)

FIGURE 1: Confocal microscopy microphotographs of non-small cell lung carcinoma. (a) FITC-labeled cytokeratin (green signal). Observe the intense reactivity of MNF116 antibody (reacts with cytokeratins 5, 6, 8, 17, and probably also 19) mainly located in the cytoplasm of malignant epithelial cells. (b) Nuclei counterstained with propidium iodide (red signal). (c) Overlay of microphotographs (a) and (b). Yellow bars = $100 \mu \mathrm{m}$.

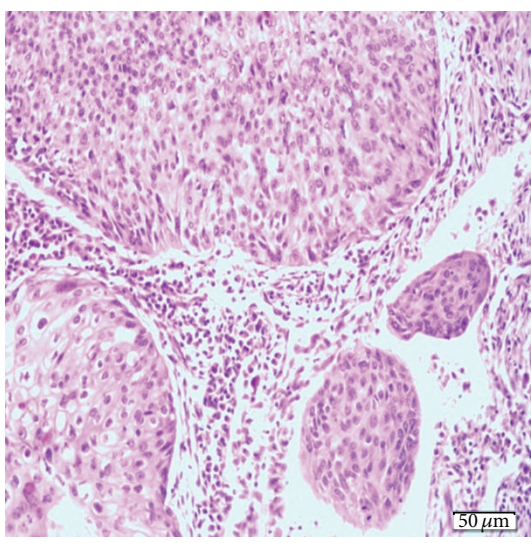

(a)

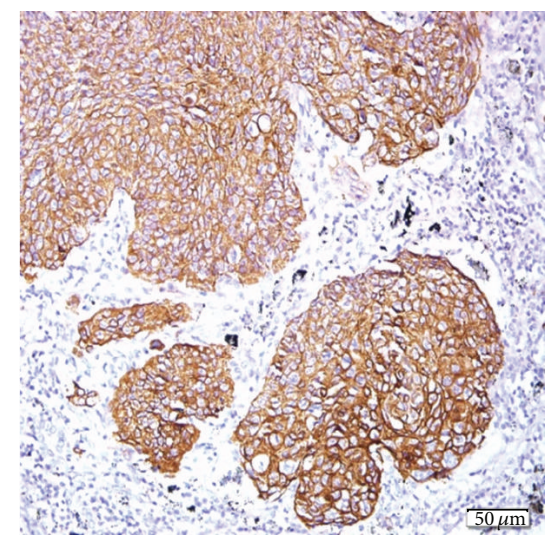

(b)

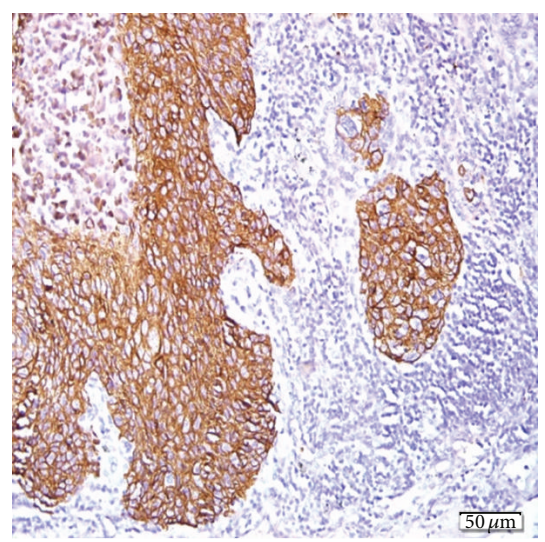

(c)

Figure 2: Microphotographs of squamous carcinoma of the lung. (a) Hematoxylin and eosin coloration. Immunohistochemical localization of cytokeratin was performed by means of MNF116 (reacts with cytokeratins 5, 6, 8, 17, and probably also 19) and 34 $\beta$ E12 antibodies (reacts with high molecular weight cytokeratins 1, 5, 10, and 14). Observe the intense reactivity of these Mabs located mainly in the cytoplasm of malignant epithelial cells. Conventional immunoperoxidase technique counterstaining with Carrazzi hematoxylin. Black bar $=50 \mu \mathrm{m}$.

TABLE 3: Expression of both high and low molecular weight cytokeratins according to histopathological type.

\begin{tabular}{lccc}
\hline Tissue samples & Number of cases & ${ }^{\mathrm{a}} 34 \beta \mathrm{E} 12+{ }^{\mathrm{b}} 35 \mathrm{BH} 11$ \\
\hline Low/high expression (\%) & & - & + \\
Epidermoid carcinoma & 16 & 56.3 & 43.7 \\
Adenocarcinoma & 20 & 80.0 & 20.0 \\
Large cell carcinoma & 10 & 50.0 & 50.0 \\
Other & 6 & 66.7 & 33.3 \\
Small cell carcinoma & 16 & 100 & 0 \\
\hline
\end{tabular}

${ }^{a} 34 \beta$ E12 reacts with high molecular weight cytokeratins (1, 5, 10, and 14); ${ }^{b} 35$ BH11 stains cytokeratin 8 (low molecular weight cytokeratin).

was scored (see Section 2.5) according to both intensity of reaction and percentage of positive cells (Figure 3 ). In the group of NSCLC patients with increased expression of high
TABLE 4: Expression of Ber-EP4 according to histopathological type.

\begin{tabular}{lccc}
\hline Tissue samples & Number of cases & \multicolumn{2}{c}{ Ber-EP4 $^{\mathrm{a}}$} \\
\hline Low/high expression (\%) & & - & + \\
Epidermoid carcinoma & 16 & 52.5 & 47.5 \\
Adenocarcinoma & 20 & 25.0 & 75.0 \\
Large cell carcinoma & 10 & 50.0 & 50.0 \\
Other & 6 & 66.7 & 33.3 \\
Small cell carcinoma & 16 & 56.3 & 43.7 \\
\hline
\end{tabular}

${ }^{a}$ Ber-EP4 antibody recognizes a transmembrane glycoprotein functioning as a cellular adhesion molecule.

molecular weight cytokeratins, the occurrence of disease recurrence $(11 / 18 ; 61.1 \%)$ was significantly more frequent than in patients with low expression of these cytokeratins $(7 / 34$; 20.6\%) $(P=0.0057$; Fisher's exact test $)$. There were no 


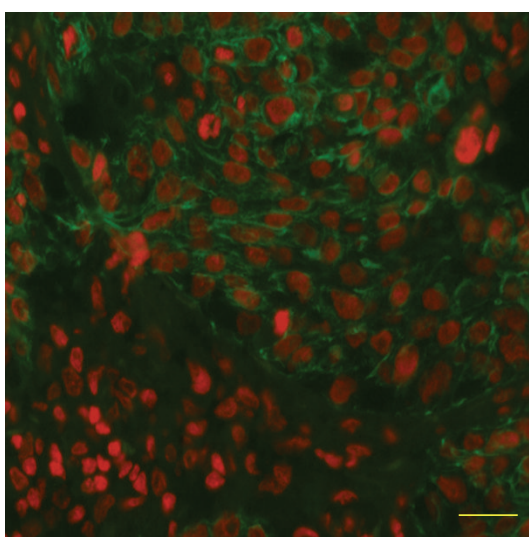

(a)

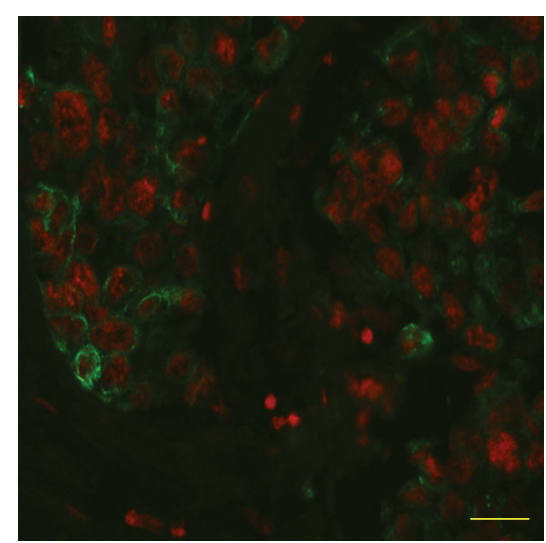

(b)

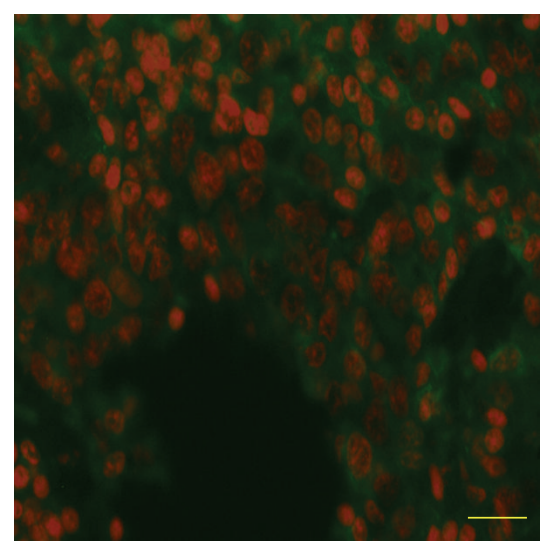

(c)

FIGURE 3: Conventional fluorescence microphotographs of non-small cell lung carcinomas. (a) Intense, (b) intense but focal, and (c) moderate and diffuse expression of high molecular weight cytokeratins (CKs 1, 5, 10, and 14) mainly located in the cytoplasm of malignant epithelial cells. FITC-labeled cytokeratin (green signal). Nuclei contrasted with propidium iodide (red signal). Yellow bars $=100 \mu \mathrm{m}$.

TABLE 5: Survival of NSCLC patients according to antigens expression profile.

\begin{tabular}{|c|c|c|c|c|}
\hline Antigens expression & $34 \beta \mathrm{E} 12^{\mathrm{a}}$ & $35 \mathrm{BH}_{11}{ }^{\mathrm{b}}$ & $\mathrm{MNF} 16^{\mathrm{c}}$ & Ber-EP4 ${ }^{\mathrm{d}}$ \\
\hline Low/high reactivity & + & + & + & + \\
\hline Number of cases & 41 & 26 & 24 & 29 \\
\hline$\% 4$-year survival rate & $87.8 \quad 27.3$ & 73.176 .9 & 66.782 .1 & $82.8 \quad 65.2$ \\
\hline $\begin{array}{l}\text { Mean survival } \\
\text { (months) }\end{array}$ & 36.029 .3 & 35.233 .9 & 32.737 .0 & $34.3 \quad 34.8$ \\
\hline$P$ value & 0.0315 & 0.8380 & 0.1340 & 0.8268 \\
\hline
\end{tabular}

${ }^{a} 34 \beta$ E12 reacts with high molecular weight cytokeratins $(1,5,10$, and 14); ${ }^{b} 35$ BH11 stains cytokeratin 8 (low molecular weight cytokeratin); ${ }^{\mathrm{c}}$ MNF116 reacts with cytokeratins $5,6,8,17$, and probably also $19 ;{ }^{\mathrm{d}}$ Ber-EP4 antibody recognizes a transmembrane glycoprotein functioning as a cellular adhesion molecule.

differences in the frequencies of low and high expression of high molecular weight cytokeratins when gender, age of patients, pathologic stage, tumor size, occurrence of lymph node and other site metastasis, histopathological type, grade of differentiation, degree of cell pleomorphism, index of tumor necrosis, and mitotic index were considered.

No correlation between low molecular weight cytokeratin and Ber-EP4 expressions and clinic-pathological features was obtained in NSCLC patients. Concerning SCLC, no association between cytokeratin and Ber-EP4 tissue expressions and clinic-pathological characteristics was evidenced.

\subsection{Tissue Expression of High Molecular Weight Cytokeratins} Was Significantly Predictive of Survival Outcome. Table 5 shows the survival distribution in NSCLC patients according to antigens expression profile. The mortality of NSCLC patients displaying high and low expression of high molecular weight cytokeratins was $19.5 \%$ and $45.5 \%$, respectively. Consequently, the expression of high molecular weight cytokeratins was found to be associated with a poor 4-year overall survival of NSCLC patients $(P=0.0315$; Log rank test $)$

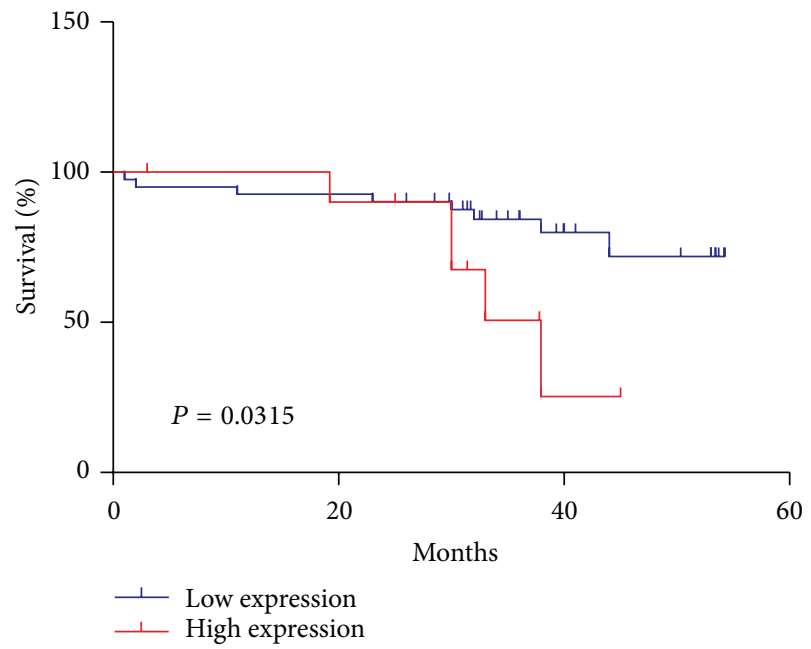

FIgURE 4: Kaplan-Meier estimate of 4-year overall survival among NSCLC patients showing different levels of high molecular weight cytokeratins expression $(P=0.0315$; Log rank test).

(Figure 4), not taking into account the histopathological classification of tumors. Similar results were obtained when 8 -year overall survival was assessed $(P=0.0103$; Log rank test) (data not shown).

Differences in the reactivity of low molecular weight cytokeratin (Figure 5), pan-cytokeratins, and Ber-EP4 monoclonal antibodies were not significantly predictive of survival outcome $(P=0.8380, P=0.1340$, and $P=0.8268$; Log rank test, resp.).

\section{Discussion}

Lung cancer is classified into small cell carcinoma (SCLC) and non-small cell carcinoma (NSCLC). The profile of molecular and genetic alterations considerably differs between SCLC and NSCLC, as well as among the subtypes of NSCLC [13]. 


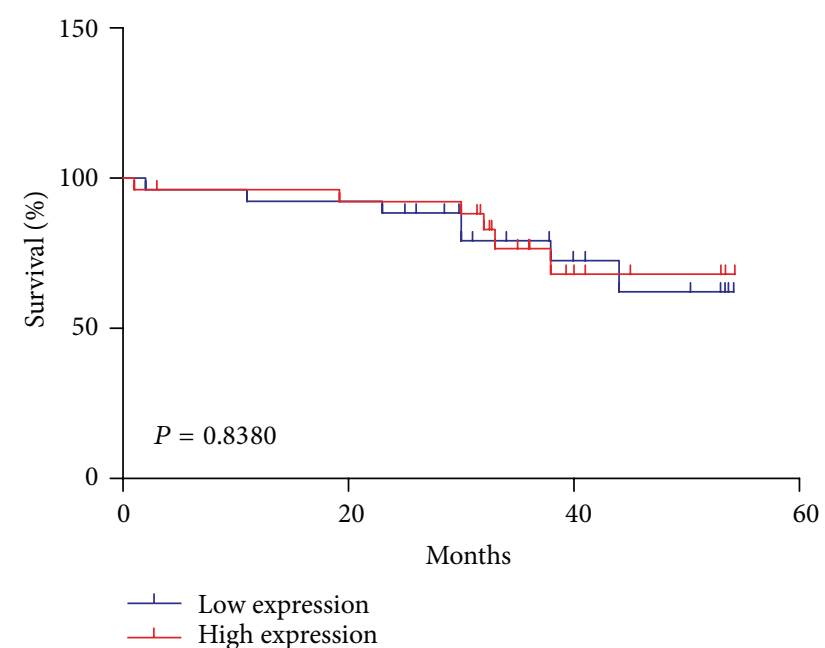

FIGURE 5: Kaplan-Meier estimate of 4-year overall survival among NSCLC patients showing different levels of low molecular weight cytokeratins expression $(P=0.8380$; Log rank test $)$.

However, SCLC is the most aggressive lung cancer and almost fatal despite a good initial response to chemoradiation therapy [14]. In spite of recent progresses in the diagnosis and treatment, the prognosis of lung cancer still remains poor $[1,2]$. A variety of prognostic factors have been identified in patients bearing lung cancer. Disease stage and performance status at time of diagnosis are considered among the most important prognostic factors in patients with NSCLC [5].

In this study, we showed an increased overall survival of patients at stages I-II as compared with the group of patients at stages III-IV, as well as in patients with absence of metastasis (lymph node and other site) and local recurrence. Concerning SCLC, patients showed increased disease recurrence, grade of tumor differentiation, and both necrosis and mitotic index displaying a poorer overall survival as compared with NSCLC. Nevertheless, the pathology-based TNM stage classification (tumor, node, and metastasis) has been considered to provide imprecise information about the survival rates [6]. Consequently, numerous studies are currently focusing in the selection of newer biological and molecular prognostic factors as a potential complement of TNM staging system [15-17].

In line with this, markers originating from the cytoskeleton, a complex network that influences the dynamic morphology of all eukaryotic cells in their tissue environment, are of great practical interest in lung cancer [18]. Among them, cytokeratins (CK), a group comprising of at least 29 different proteins, are intermediate filament proteins present in epithelial cells $[19,20]$. They are expressed in a tissuespecific manner in normal organs and the tumors that arise from them [10]. However, under the influence of intrinsic or extrinsic factors, each cell will express different types of cytokeratins in course of its evolution which is also affected by the growth and differentiation rate [21].

It is known that the immunophenotypes of the main histologic types of lung carcinoma are stable and highly reproducible. However, in the present work, it was observed as an overlapping of low and high molecular weight cytokeratins in about $30 \%$ of NSCLC samples. Between them, squamous and large cell carcinomas displayed the higher rates of cytokeratins overlapping, while the lower rates of overlapping were evidenced in adenocarcinomas. Usually, squamous cell carcinomas, independently of their site of origin, are characterized by the expression of the stratified epithelial keratins CK5, CK14, and CK17 and the hyperproliferative keratinocyte-type keratins CK6 and CK16 [9]. However, in poorly differentiated squamous cell carcinomas, coexpression of the simple epithelial keratins CK8, CK18, and CK19 is often observed [8]. Concerning large cell carcinomas, they have been considered as poorly differentiated non-small cell tumors coexpressing, in some cases, low (CK7) and high (CK5) molecular weight cytokeratins [22]. Adenocarcinomas express the simple epithelial keratins CK8, CK18, and CK19, whereas CK7 and CK20 expression is variable [9]. Because of considerable overlapping, immunophenotyping should not be used alone for histopathologic classification of lung cancer, but only as an adjunct to light microscopy [23].

Additionally, about half of the SCLC samples were stained with both pan-cytokeratins and low molecular weight (CK8) antibodies. Staining for pan-cytokeratin AE1/AE3 and low molecular weight cytokeratins (CK8, CK18, and/or CK19) antibodies has been previously demonstrated in SCLC samples $[24,25]$. However, CK7 has been considered not very useful cytokeratin for SCLC diagnosis because only about half of these tumors stain with it $[26,27]$. In line with this, in SCLC usually the diagnosis can be established based on the observation of high-quality hematoxylin and eosin stained sections without other immunostains [25]. Nevertheless, we obtained no expression of high molecular weight cytokeratins in SCLC samples. Similar results were previously published by Zhang et al. [28]. Although in the present work not so much cases were evaluated, high molecular weight keratin seems to be highly effective for distinguishing between small cell carcinoma and poorly differentiated squamous cell carcinoma.

Concerning Ber-EP4, this antigen was expressed in about $60 \%$ and $40 \%$ of NSCLC and SCLC, respectively. Malignant epithelial cells of different subtype of lung carcinoma displayed varying levels of Ber-EP4 antigen expression. But, adenocarcinomas were strongly positive ( $75 \%$ of cases) as compared with other lung carcinomas, including SCLC. Ber-EP4 antigen was reported to stain between $60 \%$ and $100 \%$ of lung adenocarcinomas [29]. In this study, the tissue expression of Ber-EP4 also correlated with the expression of the low molecular weight cytokeratin (CK8), the most common cytokeratin type found in our series of lung adenocarcinomas. Our results confirmed the increasing levels of this antigen in lung adenocarcinomas as compared with other lung tumors. The strong expression of Ber-EP4 in lung adenocarcinomas has permitted considering it a useful tool in distinguishing between these tumors and malignant mesothelioma [30, 31].

Finally, some authors have reported the prognostic value of cytokeratins in patients with non-small cell lung cancer. Nevertheless, the majority of these work included low 
molecular weight cytokeratins such as CK8 [32], CK18 [33], and CK19 [34]. Here, the reactivity of 34ßE12 antibody was grouped into low and high levels of CKs expression, following the score previously described by our group [12]. Using this score, it was found that increased expression of high molecular weight cytokeratins measured by means of $34 \beta \mathrm{E} 12$ antibody was associated with disease recurrence in NSCLC patients. Consequently, the expression of a group of high molecular weight cytokeratins by means of the clone $34 \beta$ E12 that reacts with $1,5,10$, and 14 was associated with a poorer overall survival of NSCLC, without taking into account the histopathological type.

In previous studies, the increased expression of high molecular weight CK5/6, CK14, and CK17 proteins was associated with lower grading of NSCLC [35]. However, in esophageal squamous carcinoma, the reactivity of $34 \beta E 12$ antibody correlated with the occurrence of lymph node metastasis, depth of tumor invasion, and degree of tumor differentiation [36]. The abnormal expression patterns of staining using the 34ßE12 antibody have been also related with disease recurrences and disease-free survival in patients with low-grade papillary bladder neoplasms [37] as well as an independent predictor of disease-free survival in patients with triple-negative tumors of the breast [38]. Our results could suggest the assessment of high molecular weight cytokeratins in a larger series of NSCLC samples in order to confirm the potential prognostic value of them.

\section{Conclusions}

The use of cytokeratins as diagnostic tool in tumor pathology is by far their most common application in the field of cancer. In this study, the tissue expression of low and high molecular weight cytokeratins and Ber-EP4 antigen, in both SCLC and NSCLC, was reported. Considerable overlapping of cytokeratins was evidenced in NSCLC but not in SCLC. Although in the present work a limited number of SCLC samples was evaluated, tissue expression of high molecular weight cytokeratins seems to continuously be a highly effective tool for distinguishing between SCLC and poorly differentiated squamous cell carcinoma. Additionally, the expression of high molecular weight cytokeratins was associated with disease recurrence in NSCLC patients and was significantly predictive of survival outcome. Our results could suggest the assessment of high molecular weight cytokeratins in a larger series of NSCLC samples in order to confirm the potential prognostic value of them.

\section{Conflict of Interests}

The authors declare that there is no conflict of interests regarding the publication of this paper.

\section{Authors' Contribution}

Charles E. Rengifo and Rancés Blanco contributed equally to this work.

\section{Acknowledgment}

Financial support was provided by the Center of Molecular Immunology.

\section{References}

[1] K. Kaira, N. Oriuchi, N. Sunaga, T. Ishizuka, K. Shimizu, and N. Yamamoto, "Systemic review of PET and biology in lung cancer," American Journal of Translational Research, vol. 3, no. 4, pp. 383-391, 2011.

[2] C. Gkogkou, K. Frangia, M. W. Saif, R. Trigidou, and K. Syrigos, "Necrosis and apoptotic index as prognostic factors in nonsmall cell lung carcinoma: a review," SpringerPlus, vol. 3, no. 1, pp. 1-5, 2014.

[3] G. Giaccone and P. A. Zucali, "Src as a potential therapeutic target in non-small-cell lung cancer," Annals of Oncology, vol. 19, no. 7, pp. 1219-1223, 2008.

[4] A. Barzi and N. A. Pennell, "Targeting angiogenesis in nonsmall cell lung cancer: agents in practice and clinical development," European journal of Clinical and Medical Oncology, vol. 2, no. 1, pp. 31-42, 2010.

[5] T. Berghmans, M. Paesmans, and J. P. Sculier, "Prognostic factors in stage III non-small lung cancer: a review of conventional, metabolic and new biological variables," Therapeutic Advances in Medical Oncology, vol. 3, no. 3, pp. 127-138, 2011.

[6] R.-P. Perng, C.-Y. Chen, G.-C. Chang et al., "Revisit of 1997 TNM staging system-survival analysis of 1112 lung cancer patients in Taiwan," Japanese Journal of Clinical Oncology, vol. 37, no. 1, pp. 9-15, 2007.

[7] S. Linder, "Cytokeratin markers come of age," Tumor Biology, vol. 28, no. 4, pp. 189-195, 2007.

[8] V. Karantza, "Keratins in health and cancer: more than mere epithelial cell markers," Oncogene, vol. 30, no. 2, pp. 127-138, 2011.

[9] R. Moll, M. Divo, and L. Langbein, "The human keratins: biology and pathology," Histochemistry and Cell Biology, vol. 129, no. 6, pp. 705-733, 2008.

[10] S. Kummar, M. Fogarasi, A. Canova, A. Mota, and T. Ciesielski, "Cytokeratin 7 and 20 staining for the diagnosis of lung and colorectal adenocarcinoma," British Journal of Cancer, vol. 86, no. 12, pp. 1884-1887, 2002.

[11] R. Blanco, C. E. Rengifo, M. Cedeño, M. Frómeta, E. Rengifo, and A. Carr, "Immunoreactivity of the 14F7 Mab (Raised against $\mathrm{N}$-Glycolyl GM3 ganglioside) as a positive prognostic factor in non-small-cell lung cancer," Pathology Research International, vol. 2012, Article ID 235418, 12 pages, 2012.

[12] C. E. Rengifo, R. Blanco, D. Blanco, M. Cedeño, M. Frómeta, and E. Rengifo, "Immunohistochemical characterization of three monoclonal antibodies raised against the epidermal growth factor and its receptor in non-small cell lung cancer: their potential use in the selection of patients for immunotherapy," Journal of Biomarkers, vol. 2013, Article ID 627845, 9 pages, 2013.

[13] H. Kitamura, T. Yazawa, K. Okudela, H. Shimoyamada, and H. Sato, "Molecular and genetic pathogenesis of lung cancer: differences between small-cell and non-small-cell carcinomas," The Open Pathology Journal, vol. 2, pp. 106-114, 2008.

[14] W. Travis, S. Nicholson, F. R. Hirsch et al., "Small cell carcinoma," in Tumours of the Lung. Tumours of the Lung, Pleura, Thymus and Heart. World Health Organization Classification of 
Tumours, W. D. Travis, E. Brambilla, H. K. Mueller-Hermelink, and C. C. Harris, Eds., Pathology \& Genetics, pp. 31-34, IARC Press, Lyon, France, 2004.

[15] X. Jin, Z. Wang, L. Qiu et al., "Potential biomarkers involving IKK/RelA signal in early stage non-small cell lung cancer," Cancer Science, vol. 99, no. 3, pp. 582-589, 2008.

[16] Y. Qiu, H. Yang, H. Chen et al., "Detection of CEA mRNA, p53 and AE1/AE3 in haematoxylin-eosin-negative lymph nodes of early-stage non-small cell lung cancer may improve veracity of $\mathrm{N}$ staging and indicate prognosis," Japanese Journal of Clinical Oncology, vol. 40, no. 2, Article ID hyp144, pp. 146-152, 2010.

[17] R. Blanco, C. E. Rengifo, M. Cedeño, M. Frómeta, and E. Rengifo, "Flow cytometric measurement of aneuploid DNA content correlates with high S-phase fraction and poor prognosis in patients with non-small-cell lung cancer," ISRN Biomarkers, vol. 2013, Article ID 354123, 8 pages, 2013.

[18] G. Buccheri and D. Ferrigno, "Lung tumor markers of cytokeratin origin: an overview," Lung Cancer, vol. 34, supplement 2, pp. S65-S69, 2001.

[19] G. B. Jens, S. N. Hubertus, G. Bernhard, H. Achim, and J. S. Norbert, "CK-19 serum analysis in patients with esophageal cancer," Clinical Cancer Research, vol. 6, pp. 249-252, 2000.

[20] J. Fujita, Y. Ohtsuki, S. Bandoh et al., "Elevation of cytokeratin 19 fragment (CYFRA 21-1) in serum of patients with radiation pneumonitis: possible marker of epithelial cell damage," Respiratory Medicine, vol. 98, no. 4, pp. 294-300, 2004.

[21] D. Rastel, A. Ramaioli, F. Cornillie, and B. Thirion, "CYFRA 211 , a sensitive and specific new tumour marker for squamous cell lung cancer. Report of the first European multicentre evaluation," European Journal of Cancer, vol. 30, no. 5, pp. 601606, 1994.

[22] M. Barbareschi, C. Cantaloni, V. D. Vescovo et al., "Heterogeneity of large cell carcinoma of the lung: an immunophenotypic and miRNA-based analysis," American Journal of Clinical Pathology, vol. 136, no. 5, pp. 773-782, 2011.

[23] L. Johansson, "Histopathologic classification of lung cancer: relevance of cytokeratin and TTF-1 immunophenotyping," Annals of Diagnostic Pathology, vol. 8, no. 5, pp. 259-267, 2004.

[24] J. N. Kitinya, M. Kingo, and K. Tanaka, "Expression of cytokeratin and neuron-specific enolase in small cell carcinomas of the lung," Histology and Histopathology, vol. 2, no. 4, pp. 333-343, 1987.

[25] W. D. Travis, "Update on small cell carcinoma and its differentiation from squamous cell carcinoma and other non-small cell carcinomas," Modern Pathology, vol. 25, pp. 18-30, 2012.

[26] P. Chu, E. Wu, and L. M. Weiss, "Cytokeratin 7 and Cytokeratin 20 expression in epithelial neoplasms: a survey of 435 cases," Modern Pathology, vol. 13, no. 9, pp. 962-972, 2000.

[27] M. Bobos, P. Hytiroglou, I. Kostopoulos, G. Karkavelas, and C. S. Papadimitriou, "Immunohistochemical distinction between Merkel cell carcinoma and small cell carcinoma of the lung," American Journal of Dermatopathology, vol. 28, no. 2, pp. 99104, 2006.

[28] H. Zhang, J. Liu, P. T. Cagle, T. C. Allen, A. C. Laga, and D. S. Zander, "Distinction of pulmonary small cell carcinoma from poorly differentiated squamous cell carcinoma: an immunohistochemical approach," Modern Pathology, vol. 18, no. 1, pp. 111118, 2005.

[29] C. W. Michael, "Lung Carcinoma," in Serous Effusions, B. Davidson, P. Firat, and C. W. Michael, Eds., pp. 27-45, Springer, London, UK, 2012.
[30] N. G. Ordóñez, "Value of the Ber-EP4 antibody in differentiating epithelial pleural mesothelioma from adenocarcinoma: The M.D. Anderson experience and a critical review of the literature," American Journal of Clinical Pathology, vol. 109, no. 1, pp. 85-89, 1998.

[31] I. N. Soomro, R. Oliveira, J. Ronan, Z. R. Chaudry, and J. Johnson, "Expression of mesothelial markers in malignant mesotheliomas: an immunohistochemical evaluation of 173 cases," Journal of the Pakistan Medical Association, vol. 55, no. 5, pp. 205-209, 2005.

[32] Y. Fukunaga, S. Bandoh, J. Fujita et al., "Expression of cytokeratin 8 in lung cancer cell lines and measurement of serum cytokeratin 8 in lung cancer patients," Lung Cancer, vol. 38, no. 1, pp. 31-38, 2002.

[33] R. Cohen, A. Guber, A. Siegal et al., "The prognostic application of cytokeratin typing of non small cell lung carcinoma," Cancer, vol. 79, pp. 468-473, 1997.

[34] M. Kosacka and R. Jankowska, "The prognostic value of cytokeratin 19 expression in non-small cell lung cancer," Pneumonologia i Alergologia Polska, vol. 75, no. 4, pp. 317-323, 2007.

[35] Y. Chen, T. Cui, L. Yang et al., "The diagnostic value of cytokeratin 5/6, 14, 17, and 18 expression in human non-small cell lung cancer," Oncology, vol. 80, pp. 333-340, 2011.

[36] M. Ono, H. Kijima, H. Seino, K. Hakamada, and Y. Igarashi, "Expression of cytokeratin $34 \beta \mathrm{E} 12$ is a good indicator of tumor progression in esophageal squamous cell carcinoma," Biomedical Research, vol. 33, no. 3, pp. 183-189, 2012.

[37] D. Ramos, S. Navarro, R. Villamón, M. Gil-Salom, and A. Llombart-Bosch, "Cytokeratin expression patterns in low-grade papillary urothelial neoplasms of the urinary bladder," Cancer, vol. 97, no. 8, pp. 1876-1883, 2003.

[38] R. Mehta, R. K. Jain, N. Sneige, S. Badve, and E. Resetkova, "Expression of high-molecular-weight cytokeratin (34 $\beta$ E12) is an independent predictor of disease-free survival in patients with triple-negative tumours of the breast," Journal of Clinical Pathology, vol. 63, no. 8, pp. 744-747, 2010. 

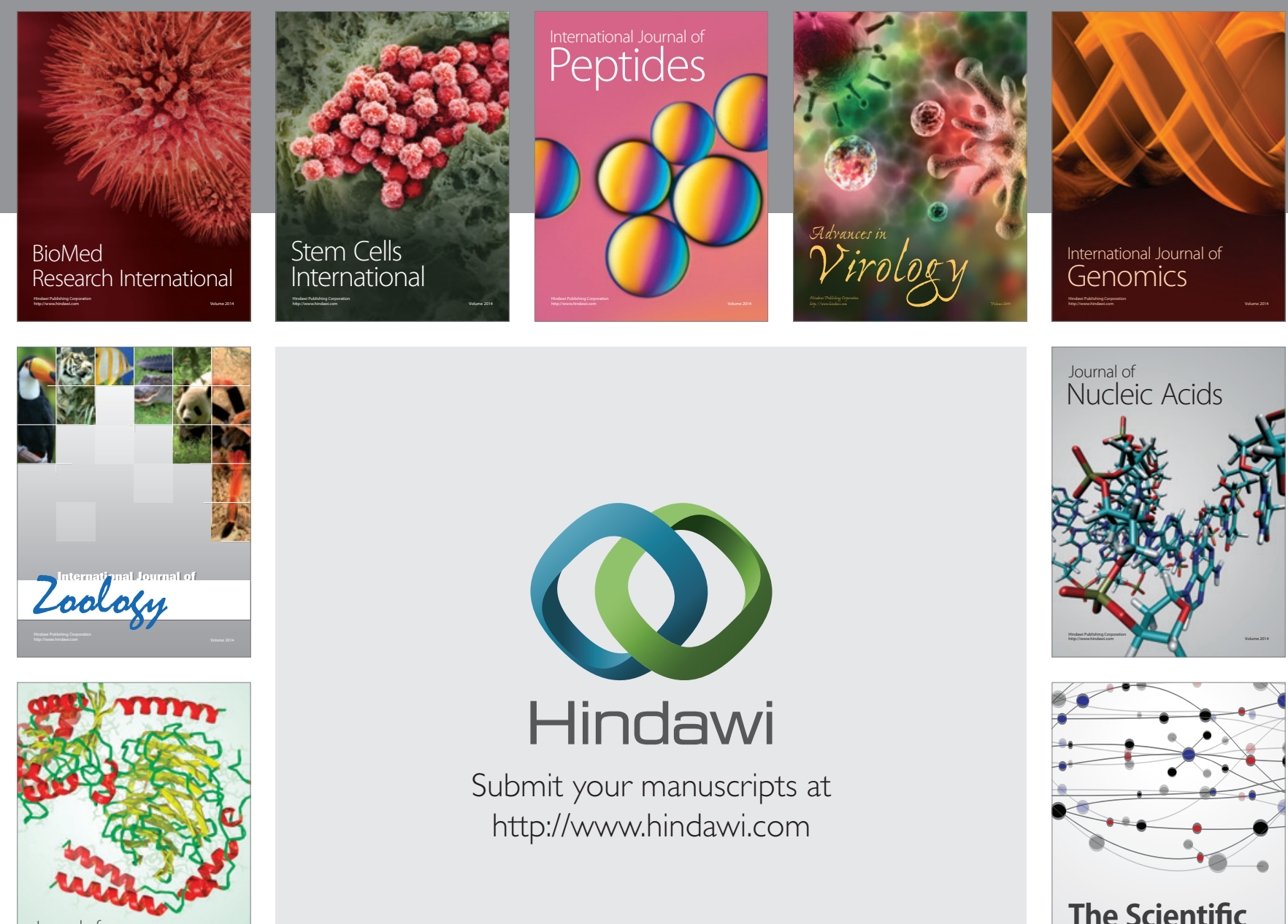

Submit your manuscripts at

http://www.hindawi.com

Journal of
Signal Transduction
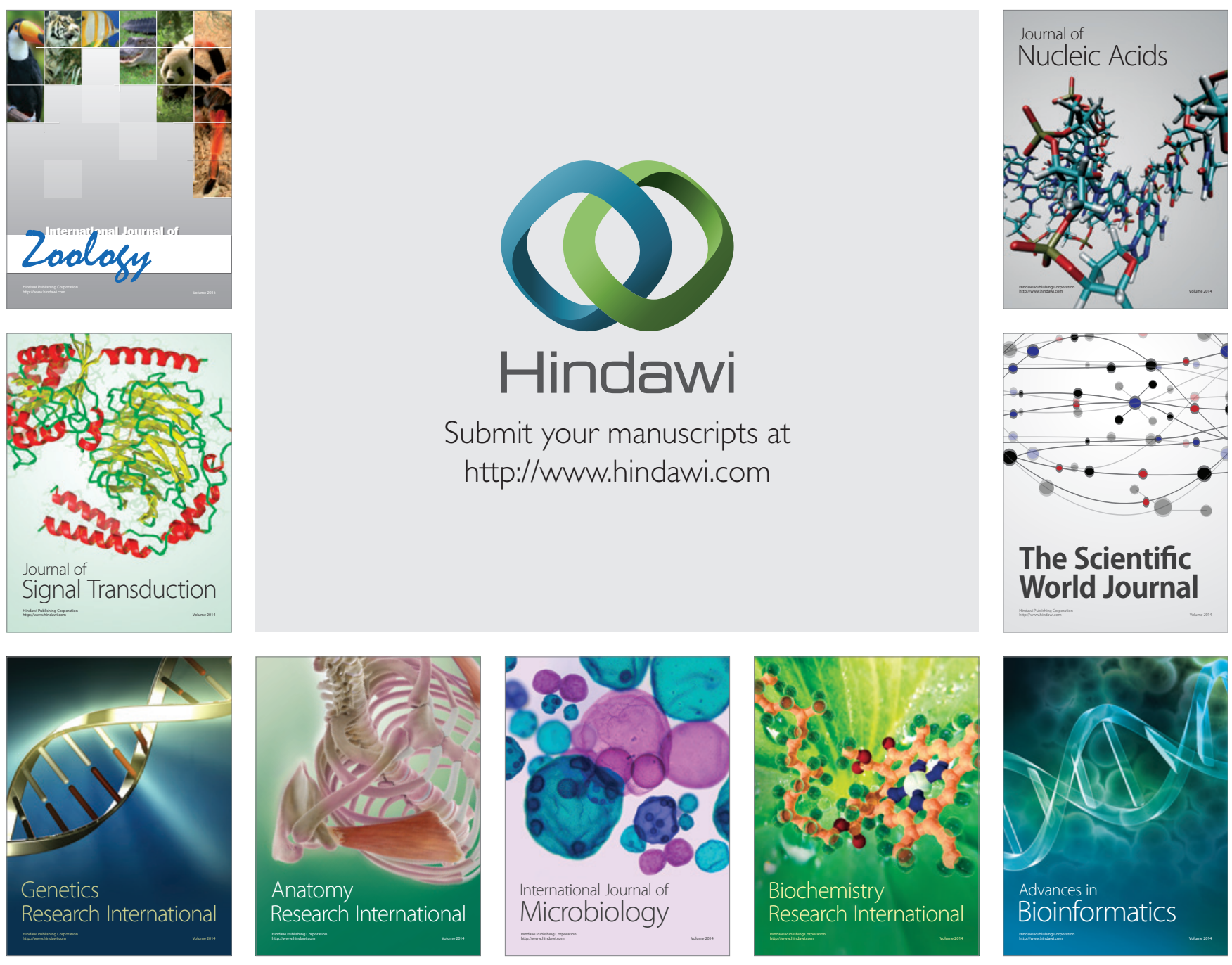

The Scientific World Journal
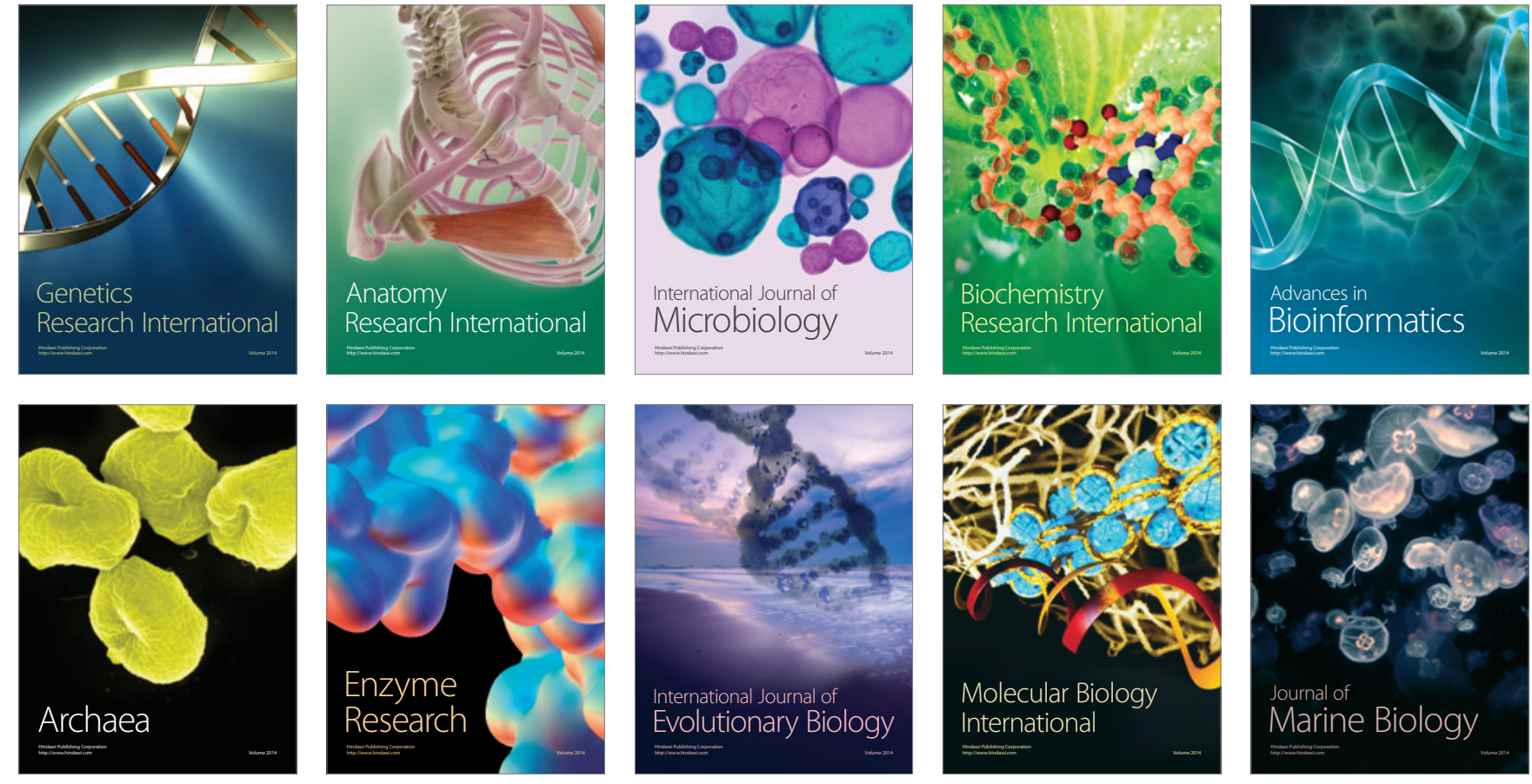